\title{
Nurse Scheduling Problem using Fuzzy Goal Programming with MINMAX Approach
}

\author{
Shely Nur Fitriani ${ }^{1,}{ }^{*}$, Bambang Irawanto ${ }^{1}$, Abdul Aziz ${ }^{1}$ \\ ${ }^{1}$ Department of Mathematics, Faculty of Science and Mathematics, Diponegoro University, Semarang, Indonesia \\ Email: shelynurfitriani@gmail.com,b_irawanto@yahoo.co.id, abdul_aziz01@yahoo.com \\ *Corresponding Author: Shely Nur Fitriani, Email: shelynurfitriani@gmail.com
}

How to cite this paper: Shely Nur Fitriani, Bambang Irawanto, Abdul Aziz (2020). Nurse Scheduling Problem using Fuzzy Goal Programming with MINMAX Approach. Journal of the Institute of Electronics and Computer, 2, 151-161. https://doi.org/10.33969/JIEC.2020.21010.

Received: Ocotober 6, 2020

Accepted: December 18, 2020

Published: December 28, 2020

Copyright ( 2020 by author(s) and Institute of Electronics and Computer. This work is licensed under the Creative Commons Attribution International License (CC BY 4.0).

http://creativecommons.org/licenses/by/4.0/

\begin{abstract}
Nurse scheduling is a complex issue since each nurse has its own work day and day off which needs to be managed. The non-singular hospital objectives, as well as the uncertainty of the objective value add to the complexity of this problem. In this article, nurse scheduling problems will be solved using Fuzzy Goal Programming with the MINMAX approach. The hospital's objectives of optimizing the number of days off for nurses, the number of working days, evening shifts, and isolated work days can be achieved. The resulting solution from the computation process meets the specified deviation tolerance limits. Problem constraints regarding requests for nurse leave, the minimum number of nurses on duty per shift, restrictions on sequential working days, and other managerial rules can also be met. With the MINMAX approach, the imbalance between positive and negative deviation values can be overcome. In addition, sensitivity analysis is also easier to be done. This article contributes to the future by revealing that the use of fuzzy set theory with unbalanced positive and negative deviations can be effectively used in nurse scheduling problems. Thus, a satisfactory schedule which can meet nurse preferences and hospital policies can be made properly.
\end{abstract}

\section{Keywords}

Fuzzy logic, Goal Programming, MINMAX Goal Programming, nurses scheduling, paid leave

\section{Introduction}

Employee scheduling problem were often found in institutes such as hospital. Hospitals are run to improve the quality and maintain health service standards. In order to achieve these goals, a hospital should own a proper scheduling system to optimize the running service. One of the main problem that should be taken seriously is Nurse Scheduling Problem (NSP). 
Nurses can be divided into 3 types, namely the head of the room, team leader, and nursing staff. Nurse scheduling then becomes a complex matter since each type of nurse needs to have their own day off and work hour. Thus, a scheduling must consider several factors, such as nurses' health, nurses' preference, and applicable managerial rules in the hospital.

The problem boundaries used in this study including requests for nurse leave, minimum number of nurses on duty per shift, maximum number of consecutive working days allowed, and applicable hospital policies. Nurse scheduling problems are formulated to maximize nurses' days off and to minimize work days, evening shifts, and isolated work days. The value of the objectives cannot be certainly determined. It was difficult for hospital management to determine the optimal number of days off and work days for nurses. To achieve a non-single goal and the uncertainty of the objective value, this problem can be solved by using the Fuzzy Goal Programming (FGP) model [1]-[3]. In the FGP model, the value of the positive deviation and the negative deviation is determined for each goal. These deviation values become the allowable limit for the deviation of the target value.

In this article, nurse scheduling problem will be solved using FGP model with MINMAX approach [4]. This approach applies conventional MINMAX Goal Programming (MGP) model [5] into FGP problems. A similar study which applied MINMAX approach into FGP in NSP has yet to be found. MINMAX approach deals with unbalanced value of positive deviation and negative deviation. In addition, a sensitivity analysis could be easier done. In FGP, the decision maker cannot determine the objective value precisely, thus changes in the value of tolerance for deviation may occur frequently. With the MINMAX approach, the sensitivity analysis is carried out only by changing the maximum value of positive and negative deviation variables in the model. On the other hand, a recalculation of all constraints is necessary towards the sensitivity analysis on the conventional FGP model.

The structure of this article is as follows. A literature study is provided in section 2. Section 3 presents an illustration of the problem, how the formulation uses the proposed model, and the resulting computational output. A conclusion and how this article will contribute to further research are presented in section 4.

\section{Related Literature}

Nurse Scheduling Problem (NSP) has became an interesting topic many people had studied. One of its simplest method was to use a linear programming. A study using conventional linear programming was done by Kumar and Nagalakshmi [6], in which they proposed only one objective function, namely to minimize the number of 
Shely Nur Fitriani et al.

working nurse per day. Smet, et al [7], used an integer programming and introduced the concept of local and global consistency in the constraint evaluation process. In another study, Mujahid, et al [8] used an integer programming in the form of binary (0-1). Trilling, et al [9] suggested two methods to solve the scheduling problem for anesthetic nurse, that are integer programming and constraint programming.

Millar and Kiragu [10] provided a mathematical model for the cyclic and non-cyclic scheduling of a 12-hour-shift nurse. They introduced a concept called stint, which was a pattern characterized by start date, duration, cost, and work shift. Acyclic graph in determining the nurses' schedule was created by using the stint as a node in the network. The resulting model was the shortest path problem with side constraints. The problems in cyclic and non-cyclic scheduling could be solved with a little modification in the network used. Purnomo and Bard [11] merged elements of cyclic scheduling and preference scheduling by developing a branch-and-price algorithm which uses several branching rules and rounding heuristics.

Moz and Pato [12] tried to solve nurse re-scheduling problems which occur when a nurse cannot attend the work as scheduled. They presented two new integer multicommodity flow formulations for the re-scheduling problems, namely using a directed multilevel acyclic network and node aggregation.

Topolaglu and Selim [13] applied fuzzy set theory to a multi-objective integer programming model to overcome the uncertainty of hospital management target values and nurse preference. They presented three different fuzzy solution approaches, i.e. Belmann and Zadeh's min operator [14], Werner's-fuzzy-and operator [15], and Li's two-phase approach [16]. Cetin and Sarucan [17] proposed multi-objective integer programming model in dealing with NSP problems. A programming approach using fuzzy Chang [18] objectives was then conducted. Bagheri, Devin and Izanloo [19] suggested a stochastic optimization model, in which they calculated the uncertainties in the demand and stay period of patients over time. In order to obtain an optimal schedule, Sample Average Approximation approach was used.

\section{Nurse Scheduling Problem}

A room in a hospital has 18 nurses, including 1 head of the room, 3 team leaders, and 14 nursing staffs. In this article, the author will formulate a schedule for nurses in the room on September 2019, which consists of 30 days. There are six work shifts in this room, i.e day off, morning shift (07.00-14.00 WIB), afternoon shift (14.00-21.00 WIB), evening shift (21.00-07.00 WIB), supervision (07.00-15.00 WIB), and paid leave. Supervision were handled by head of the room and team leader I. 
Each day, a nurse cannot afford to get more than 1 shift. Head of the room and team leader only works in morning shift, unless there are supervision duties. Head of the room gets a supervision shift on 7th and 27th of September, while team leader gets a shift on 8th and 22nd of September. Nurses can afford random shifts from morning to evening. Head of the room only does shifts until Friday, and they should take days off on Saturday and Sunday, unless, again, if there are supervision duties. Team leaders was off every Sunday, except for team leader I when supervision shift is needed. Every morning but on Sunday, at least there is 1 team leader on duty.

Each nurse is not allowed to work more than 6 consecutive days, to get 2 evening shifts in row, to get a morning or afternoon shift after getting evening shift, to get a morning shift after afternoon shift, and to get an evening shift after morning shift. There should be at least 5 nurses in morning shift, excluding the head of the room for he works separately from the patients' ward. Three or four nurses are needed specifically on Sunday, since there are usually less people than other days. For each afternoon and evening shift, minimum of 3 and maximum of 4 nurses are needed. In order to maintain work's efficiency, the hospital management does not allow an off-on-off work pattern and asks to avoid on-off-on pattern for day off as possibly can.

There are 6 nurses requesting paid leave in this month. Team leader III asked for leave on September 3 and 4. Nurse 7 asked for leave on September 20, 21, and 22. Nurse 10 and nurse 15 both asked for leave on September 3, 4, and 5 . Nurse 12 and nurse 17 asked for leave on September 8, 9, and 10. The hospital management intends to create a schedule in which the on-off-on pattern is avoided in order to maintain the nurses' efficiency.

The index and variables used are:

$a=$ nurse index (where $a=1$ is the head of the room, $a=2,3,4$ is team leaders, and $a=5,6, \ldots, 18$ is nursing staff)

$b \quad=$ index of days in a scheduling period $(b=1,2, \ldots, 30)$

$c \quad$ = shift index, with $c=1,2,3,4,5,6$

( 1 = day off, $2=$ morning shift, $3=$ afternoon shift, $4=$ evening shift, 5 =supervision, 6 =paid leave)

$d u r_{c}=$ duration of shift $c$, with $d u r_{1}=0, d u r_{2}=7, d u r_{3}=7, d u r_{4}=10$, $d u r_{5}=8$ dan $d u r_{6}=0$

Since the decisions taken are in the form of 'yes' or 'no', the decision variables of this problem are binary variable, namely:

$$
X_{a b c}= \begin{cases}1, & \text { if nurse } a \text { on day } b \text { gets shift } c \\ 0, & \text { others }\end{cases}
$$

This scheduling problem will be solved using FGP with MINMAX approach. 
Shely Nur Fitriani et al.

\subsection{Problem Formulation}

\section{Objective function}

The objective function of this problem is to maximize the degree of achievement of the overall goal, which is represented by using auxiliary variables $(\lambda)$

$$
\max \lambda
$$

$\lambda=$ degree of achievement

\section{Goal constraint}

The least working hours for nurses who do not take any leave are 155 hours, 140 hours for nurses who take 2 days leave, and 135 hours for nurses who take 3 days leave.

$$
\begin{aligned}
& f_{1_{a}}(x)+n_{1_{a}}-p_{1_{a}}=155, \text { for } a \neq 4,7,10,12,15,17 \\
& f_{1_{a}}(x)+n_{1_{a}}-p_{1_{a}}=140 \text {, for } a=4 \\
& f_{1_{a}}(x)+n_{1_{a}}-p_{1_{a}}=135 \text {, for } a=7,10,12,15,17 \\
& \text { with } \\
& f_{1_{a}}=\text { working hours of nurse }-a \\
& n_{1_{a}}=\text { negative deviation of nurse's working hours } \\
& p_{1_{a}}=\text { positive deviation of nurse's working hours }
\end{aligned}
$$

Nurses receive at least 9 days off a month

with

$$
f_{2_{a}}(x)+n_{2_{a}}-p_{2_{a}}=9, \text { for } a=1,2, \ldots, 18
$$

$f_{2_{a}}=$ days off of nurse $-a$

$n_{2_{a}}=$ negative deviation of nurse's day off

$p_{2_{a}}=$ positive deviation of nurse's day off

Nurses cannot get more than 6 evening shifts in a month

$$
f_{3_{a}}(x)-p_{3_{a}} \leq 6 \text {, for } a=1,2, \ldots, 18
$$

with

$f_{3_{a}}=$ Number of night shift for nurse $-a$

$p_{3 a}=$ positive deviation of nurse's evening shift

On-off-on patterns are avoided as possibly can

$$
\begin{aligned}
& \quad f_{4 a b}(x)-p_{4_{a b}} \leq 2 \text {, for } a=1,2, \ldots, 18 \text { and } b=2,3, \ldots, 29 \\
& \text { with } \\
& f_{4_{a b}}(x)=\sum_{c=2}^{5} X_{a(b-1) c}+\left(X_{a b 1}+X_{a b 6}\right)+\sum_{c=2}^{5} X_{a(b+1) c}
\end{aligned}
$$

\section{Fuzzy Constraint}

The maximum deviation of working hours is 11 hours, either above or below the target value

$$
\lambda+\frac{1}{11} n_{1 a}+\frac{1}{11} p_{1 a} \leq 1, \text { for } a=1,2, \ldots, 18
$$


The maximum deviation of day off is 3 days, either above or below the target value

$$
\lambda+\frac{1}{3} n_{2_{a}}+\frac{1}{3} p_{2 a} \leq 1, \text { for } a=1,2, \ldots, 18
$$

The maximum number of night shift deviation is 2 times above the target value

$$
\lambda+\frac{1}{2} p_{3 a} \leq 1, \text { for } a=1,2, \ldots, 18
$$

The maximum on-off-on pattern deviation is 2 above the target value

$$
\lambda+\frac{1}{2} p_{4 a b} \leq 1 \text {, for } a=1,2, \ldots, 18 \text { and } b=1,2, \ldots, 30
$$

\section{System constraint}

Each nurse can only get 1 shift per day

$$
\sum_{c=1}^{6} X_{a b c}=1 \text {, for } a=1,2, \ldots, 18 \text { and } b=1,2, \ldots, 30
$$

Head of the room gets supervision on the $7^{\text {th }}$ and $27^{\text {th }}$

$$
X_{1 b 5}=1 \text {, for } b=7,27
$$

Team Leader I gets supervision on the $8^{\text {th }}$ and $22^{\text {nd }}$

$$
X_{2 b 5}=1 \text {, for } b=8,22
$$

Team Leader III asked for leave on the $3^{\text {rd }}$ and $4^{\text {th }}$

$$
X_{4 b 6}=1 \text {, for } b=3,4
$$

Every day, team leader only gets a morning shift or a day off, except when they get supervision duty or ask for leave

$$
\begin{gathered}
X_{1 b 1}+X_{1 b 2}=1 \text {, for } b \neq 7, b \neq 27 \\
X_{2 b 1}+X_{2 b 2}=1 \text {, for } b \neq 8, b \neq 22 \\
X_{3 b 1}+X_{3 b 2}=1 \text {, for } b=1,2, \ldots, 30 \\
X_{4 b 1}+X_{4 b 2}=1 \text {, for } b \neq 3 \text { dan } b \neq 4
\end{gathered}
$$

The $7^{\text {th }}$ nurse asks for leave for the $20^{\text {th }}, 21^{\text {st }}$, and $22^{\text {nd }}$

$$
X_{7 b 6}=1 \text {, for } b=20,21,22
$$

The $10^{\text {th }}$ and $15^{\text {th }}$ nurses request for leave for the $3^{\text {rd }}, 4^{\text {th }}$, and $5^{\text {th }}$

$$
X_{a b 6}=1 \text {, for } a=10,15 \text { dan } b=3,4,5
$$

The $12^{\text {th }}$ and $17^{\text {th }}$ nurses request for leave for the $8^{\text {th }}, 9^{\text {th }}$, and $10^{\text {th }}$

$$
X_{a b 6}=1 \text {, for } a=12,17 \text { and } b=8,9,10
$$

Every day, a nursing staff can get a day off, a morning, afternoon, or evening shift, except when on leave

$$
\begin{aligned}
& \sum_{c=1}^{4} X_{a b c}=1, \text { for } a=5,6, \ldots, 18 \text { and } a \neq 7,10,12,15,17 \\
& b=1,2, \ldots, 30 \\
& \sum_{c=1}^{4} X_{a b c}=1 \text {, for } a=7 \text { and } b \neq 20,21,22 \\
& \sum_{c=1}^{4} X_{a b c}=1 \text {, for } a=10,15 \text { and } b \neq 3,4,5 \\
& \sum_{c=1}^{4} X_{a b c}=1 \text {, for } a=12,17 \text { and } b \neq 8,9,10
\end{aligned}
$$

Every Saturday and Sunday the head of the room must be off, except when on supervision duty

$$
X_{1 b 1}=1 \text {, for } b=1,8,14,15,21,22,28,29
$$

Team leader must take a day off every Sunday, except for the team leader I when on supervision duty 


$$
\begin{gathered}
X_{2 b 1}=1 \text {, for } b=1,15,29 \\
X_{a b 1}=1, \text { for } a=3,4 \text { and } b=1,8,15,22,29
\end{gathered}
$$

Every morning there is at least 1 team leader on duty (except Sundays, and excluding the head of the room)

$$
\sum_{a=2}^{4} X_{a b 2} \geq 1, \text { for } b \neq 1,8,15,22,29
$$

Minimum of 5 nurses are on morning shift (excluding the head of the room), except on Sundays (minimum of 3 and less than 5 nurses))

$$
\begin{aligned}
& \sum_{a=2}^{18} X_{a b 2} \geq 5, \text { for } b \neq 1,8,15,22,29 \\
& \sum_{a=2}^{18} X_{a b 2} \geq 3, \text { for } b=1,8,15,22,29 \\
& \sum_{a=2}^{18} X_{a b 2}<5, \text { for } b=1,8,15,22,29
\end{aligned}
$$

Minimum of 3 and less than 5 nurses are needed on afternoon and evening shifts

$$
\begin{aligned}
& \sum_{a=2}^{18} X_{a b c} \geq 3, \text { for } b=1,2, \ldots, 30 \text { and } c=3,4 \\
& \sum_{a=2}^{18} X_{a b c}<5, \text { for } b=1,2, \ldots, 30 \text { and } c=3,4
\end{aligned}
$$

Nurses may not work for more than 6 consecutive days

$$
\sum_{c=2}^{5}\left(X_{a b c}+X_{a(b+1) c}+X_{a(b+2) c}+X_{a(b+3) c}+X_{a(b+4) c}+X_{a(b+5) c}+\right.
$$

$\left.X_{a(b+6) c}\right) \leq 6$, for $a=1,2, \ldots, 18$ and $b=1,2, \ldots, 24$

Nurses may not have evening shifts for more than 2 consecutive days

$X_{a b 4}+X_{a(b+1) 4}+X_{a(b+2) 4} \leq 2$, for $a=1,2, \ldots, 18$ and $b=1,2, \ldots, 28$

After getting a evening shift, nurses are not allowed to get morning or afternoon shifts on the following day

$$
X_{a b 4}+X_{a(b+1) 2}+X_{a(b+1) 3} \leq 1 \text {, for } a=1,2, \ldots, 18 \text { and } b=1,2, \ldots, 29
$$

After getting an afternoon shift, nurses are not allowed to get morning shift on the following day

$$
X_{a b 3}+X_{a(b+1) 2} \leq 1 \text {, for } a=1,2, \ldots, 18 \text { and } b=1,2, \ldots, 29
$$

In order to get morning-afternoon-evening shifts, nurses cannot get evening shifts after getting morning shifts

$$
X_{a b 2}+X_{a(b+1) 4} \leq 1, \text { for } a=1,2, \ldots, 18 \text { and } b=1,2, \ldots, 29
$$

There should be no off-on-off patterns

$$
\begin{aligned}
& \left(X_{a(b-1) 1}+X_{a(b-1) 6}\right)+\sum_{c=2}^{5} X_{a b c}+\left(X_{a(b+1) 1}+X_{a(b+1) 6}\right) \leq 2 \quad, \quad \text { for } \\
& a=1,2, \ldots, 18 \text { dan } b=2,3, \ldots, 29
\end{aligned}
$$

\section{Non-negative constraint}

$$
\lambda \geq 0
$$

$X_{a b c}=\{0,1\}$, for all $a=1,2, \ldots, 18, b=1,2, \ldots, 30$, and $c=1,2, \ldots, 6$ (43)

$n_{1_{a}}, p_{1_{a}}, n_{2_{a}}, p_{2_{a}}, p_{3 a^{\prime}}, p_{4 a b} \geq 0$, for all $a=1,2, \ldots, 18$ and $b=1,2, \ldots, 30$

\subsection{Solutions}

The formulation above was done using LINGO 18.0 software. The computational 
Table 2. objective value and their deviations

\begin{tabular}{cccccccccccc}
\hline Nurse $(a)$ & $f_{1_{a}}$ & $g_{1 a}$ & $n_{1}$ & $p_{1}$ & $f_{2_{a}}$ & $g_{2_{a}}$ & $n_{2}$ & $n_{2}$ & $f_{3_{a}}$ & $g_{3_{a}}$ & $p_{3}$ \\
\hline 1 & 156 & 155 & & 1 & 8 & 9 & 1 & & 0 & 6 & \\
2 & 156 & 155 & & 1 & 8 & 9 & 1 & & 0 & 6 & \\
3 & 154 & 155 & 1 & & 8 & 9 & 1 & & 0 & 6 & \\
4 & 140 & 140 & & & 8 & 9 & 1 & & 0 & 6 & \\
5 & 158 & 155 & & 3 & 10 & 9 & & 1 & 6 & 6 & \\
6 & 161 & 155 & 6 & 10 & 9 & & 1 & 7 & 6 & 1 \\
7 & 137 & 135 & 2 & 10 & 9 & & 1 & 6 & 6 & \\
8 & 161 & 155 & 6 & 10 & 9 & & 1 & 7 & 6 & 1 \\
9 & 158 & 155 & 3 & 10 & 9 & & 1 & 6 & 6 & \\
10 & 140 & 135 & 5 & 10 & 9 & & 1 & 7 & 6 & 1 \\
11 & 161 & 155 & 6 & 10 & 9 & & 1 & 7 & 6 & 1 \\
12 & 140 & 135 & 5 & 10 & 9 & & 1 & 7 & 6 & 1 \\
13 & 161 & 155 & 6 & 10 & 9 & & 1 & 7 & 6 & 1 \\
14 & 161 & 155 & 6 & 10 & 9 & & 1 & 7 & 6 & 1 \\
15 & 137 & 135 & 2 & 10 & 9 & & 1 & 6 & 6 & \\
16 & 155 & 155 & & & 10 & 9 & & 1 & 5 & 6 & \\
17 & 140 & 135 & 5 & 10 & 9 & & 1 & 7 & 6 & 1 \\
18 & 158 & 155 & 3 & 10 & 9 & & 1 & 6 & 6 & \\
\hline
\end{tabular}

\section{Conclusion}

Nurse scheduling arrangements usually consists of several objectives to be achieved at once. The value of each objective cannot be certainly determined. With Fuzzy Goal Programming application using the MINMAX approach, the multi-objective nurse scheduling problem with uncertain value in this article can be solved. Tolerance limits have been set for each objective, and the computation results show that the solution is within the interval of allowable tolerance. Constraints regarding requests for nurse leave, the minimum number of nurses on duty per shift, the number of consecutive working days allowed, and hospital policies can be met properly. The sensitivity analysis can be done easily by changing the maximum deviation value in equations (8)-(11).

This study contributes to the future by revealing that the use of fuzzy set theory with unbalanced positive and negative deviation can be effectively used to solve nurse scheduling problem. The resulting schedule is quite satisfying because it can meet nurse preferences and hospital policies. In the further studies, this model is expected to be developed with more complex problem constraints by adjusting to existing policies. This model is also expected to be developed into user-friendly software so that the process of decision making can be carried out faster by anyone.

\section{Conflicts of Interest}

There is no conflict of interest. 


\section{References}

[1] R. Narasimhan, "GOAL PROGRAMMING IN A FUZZY ENVIRONMENT,” Decis. Sci., vol. 11, no. 2, pp. 325-336, Apr. 1980.

[2] R. N. Tiwari, S. Dharmar, and J. R. Rao, "Fuzzy goal programming - An additive model,” Fuzzy Sets Syst., vol. 24, no. 1, pp. 27-34, 1987.

[3] Y. Kara, T. Paksoy, and C. Ter Chang, "Binary fuzzy goal programming approach to single model straight and U-shaped assembly line balancing," Eur. J. Oper. Res., vol. 195, no. 2, pp. 335-347, Jun. 2009.

[4] M. A. Yaghoobi and M. Tamiz, "A method for solving fuzzy goal programming problems based on MINMAX approach," vol. 177, pp. 1580-1590, 2007.

[5] R. Flavell, “A new goal programming formulation,” Omega, vol. 4, no. 6, pp. 731-732, 1976.

[6] B. S. Kumar, M. G. Nagalakshmi, and S. Kumaraguru, "A Shift Sequence for Nurse Scheduling Using Linear Programming Problem,” vol. 3, no. 6, pp. 24-28, 2014.

[7] P. Smet, F. Salassa, and G. Vanden Berghe, "Local and global constraint consistency in personnel rostering," Int. Trans. Oper. Res., vol. 24, no. 5, pp. 1099-1117, Sep. 2017.

[8] N. Fan et al., "Nurse scheduling problem: An integer programming model with a practical application,” Springer Optim. Its Appl., vol. 74, pp. 65-98, 2013.

[9] L. Trilling, A. Guinet, and D. Le Magny, "NURSE SCHEDULING USING INTEGER LINEAR PROGRAMMING AND CONSTRAINT PROGRAMMING,” IFAC Proc. Vol., vol. 39, no. 3, pp. 671-676, Jan. 2006.

[10] H. H. Millar and M. Kiragu, "Cyclic and non-cyclic scheduling of $12 \mathrm{~h} \mathrm{shift}$ nurses by network programming," Eur. J. Oper. Res., vol. 104, no. 3, pp. 582-592, Feb. 1998.

[11] H. W. Purnomo and J. F. Bard, "Cyclic preference scheduling for nurses using branch and price,” Nav. Res. Logist., vol. 54, no. 2, pp. 200-220, Mar. 2007.

[12] M. Moz and M. V. Pato, "Solving the problem of rerostering nurse schedules with hard constraints: New multicommodity flow models," Ann. Oper. Res., vol. 128, no. 1-4, pp. 179-197, Apr. 2004.

[13] S. Topaloglu and H. Selim, "Nurse scheduling using fuzzy modeling approach,” Fuzzy Sets Syst., vol. 161, no. 11, pp. 1543-1563, Jun. 2010.

[14] R. E. Bellman and L. A. Zadeh, "Decision-Making in a Fuzzy Environment," Manage. Sci., vol. 17, no. 4, p. B-141-B-164, Dec. 1970.

[15] B. M. Werners, "Aggregation Models in Mathematical Programming,” in Mathematical Models for Decision Support, Springer Berlin Heidelberg, 1988, pp. 295-305.

[16] R.J.Li, "Multiple objective decision making in a fuzzy environment," Kansas State University, 1990.

[17] E. Çetin and A. Sarucan, "Nurse scheduling using binary fuzzy goal 
Shely Nur Fitriani et al.

programming,” 6th Int. Conf. Model. Simulation, Appl. Optim. ICMSAO 2015 - Dedic. to Mem. Late Ibrahim El-Sadek, no. May 2015, 2015.

[18] C. Ter Chang, "Binary fuzzy goal programming," Eur. J. Oper. Res., vol. 180, no. 1, pp. 29-37, Jul. 2007.

[19] M. Bagheri, A. Gholinejad Devin, and A. Izanloo, “An application of stochastic programming method for nurse scheduling problem in real word hospital,” Comput. Ind. Eng., vol. 96, pp. 192-200, 2016. 